\title{
Mathematical model of the modified tissue deformation under stretching
}

\author{
Rashit Kayumov ${ }^{1,2}$ [0000-0003-0711-9429], and Inzilija Mukhamedova ${ }^{1 *}$ [0000-0001-5869-6686] \\ ${ }^{1}$ Kazan State University of Architecture and Engineering, 420043, Zelenaya st. Kazan, Russia \\ ${ }^{2}$ Kazan National Research Technical University named after A.N. Tupolev, 420111, K. Marks st., \\ Kazan, Russia
}

\begin{abstract}
One of the effective methods for modifying natural and synthetic materials is a use of the flocking process. To analyze a quality of the modified fabrics, it is useful to have mathematical models describing a stress-strain state of the fabrics when exposed to various loads. A method has been developed for determining the stiffness characteristics of a flocked fabric based on the results of testing samples cut at different angles to the base at different tensile forces. This technique makes it possible to analyze the effect of flocking on the mechanical characteristics of the fabric. It was revealed that the theory of mixtures, when averaging the properties of the fabrics and glue with respect to thickness, does not allow determining the stiffness characteristics with acceptable accuracy. The limits of applicability of the theory of mixtures were determined when carrying out averaging of the mechanical characteristics with respect to the area of the flocked fabrics.
\end{abstract}

Keywords: nonlinearly elastic model, flocked fabric, identification, mechanical characteristics, methodology, numerical experiment.

\section{Introduction}

Giving the improved mechanical and technological properties to the materials is carried out by means of their mechanical, chemical, electromechanical and electrophysical modification. Currently, the textile industry is increasingly using the modification methods by processing fabrics with cold plasma [1,2]. Plasma allows us to change properties of the surfaces of the materials in a wide range, improves the adhesive properties of the textile material, increases wear resistance while maintaining the previously obtained strength characteristics [3-6]. An increase in the fire resistance of linen fabrics produced by using the modification with the use of a plasma flow allows conducting a more efficient and more uniform solution absorption of the surface of textile materials [7].

A sol-gel technology is widely used for innovative finishing of textile materials [8]. The main advantage of the sol-gel method over the others is that it allows us to control the structure of the resulting materials, a size of particles, a size and volume of pores, a surface area of films in order to obtain a material with the desired properties $[9,10]$. In the modern context the researches are actively developing regarding improving the methods of

\footnotetext{
*Corresponding author: Muhamedova-inzilija@mail.ru
} 
modifying cellulose fibers to create a wide range of new materials with biocidal properties [11-13]. In the works [14-16] an antibacterial finish of textile materials was studied by copper nanoparticles.

One of the effective methods for modifying natural and synthetic materials is a use of the flocking process [17]. With this technology, it is possible to obtain the required technological, operational properties of textile materials, as well as targeted improvement in the physicomechanical properties of fabricss [18-20]. A flocated surface, while frictioning, prevents mechanical damage, which is used in the manufacture of packaging and sealing elements, such as a window profile in cars.

To analyze a quality of the modified fabrics, it is useful to have mathematical models describing a stress-strain state (SSS) of the fabrics when exposed to various loads. This is relevant due to the fact that such models will make it easier to solve the problem of optimizing a fabrics treatment technology.

\section{Materials and methods}

\subsection{Nonlinearly elastic model of strain of the fabrics}

Let us introduce the efforts per unit length of the section of our sample. They are denoted through $N_{11}, N_{22}, N_{12}$, (efforts along the basics, the weft and the shear force). The corresponding deformations are denoted through $\varepsilon_{11}, \varepsilon_{22}, \gamma_{12}$. Let us introduce the vectors $\{N\},\{\varepsilon\}$ :

$$
\{\mathrm{N}\}=\left\{N_{11}, N_{22}, N_{12}\right\}^{T} \quad\{\varepsilon\}=\left\{\varepsilon_{11}, \varepsilon_{22}, \gamma_{12}\right\}^{T} .
$$

The index $« T \gg$ means a transposition operation.

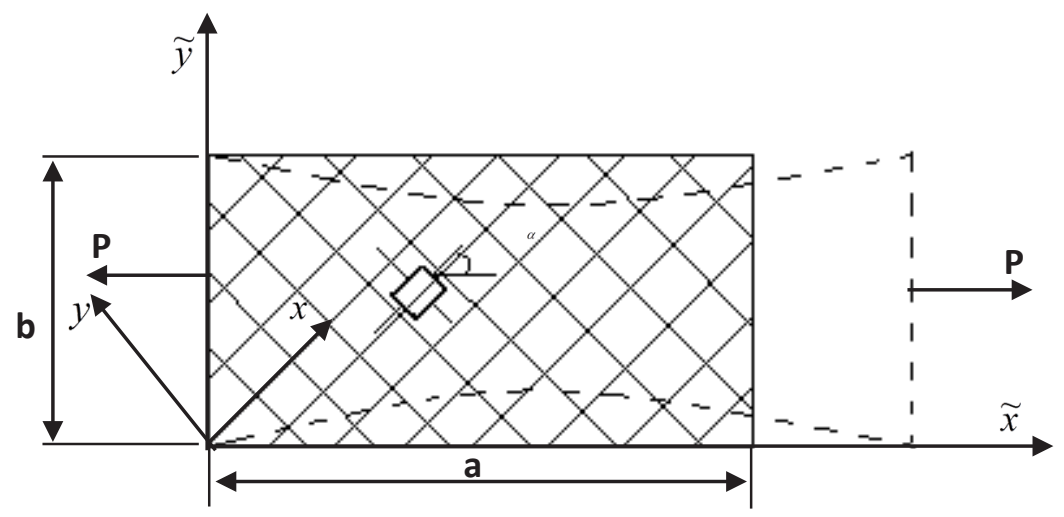

Fig. 1. Strain of the tissue sample, chipped out at an angle to the base under an action of the force P.

For a nonlinear case in the axes of orthotropy, the elastic potential for the non-flocked fabrics is taken in the following form:

$$
\begin{aligned}
& W=D_{110} \varepsilon_{11}^{2} / 2+D_{112} \varepsilon_{11}^{4} / 12+D_{120} \varepsilon_{11} \varepsilon_{22}+ \\
& +D_{220} \varepsilon_{22}^{2} / 2+D_{222} \varepsilon_{22}^{4} / 12+D_{330} \gamma_{12}^{2} / 2+D_{334} \gamma_{12}^{6} / 30 .
\end{aligned}
$$

Similarly, an elastic potential for the flocked fabrics is introduced. Let us distinguish the relations using the index «flock»: 


$$
\begin{aligned}
& W^{\text {flock }}=D_{110}^{\text {flock }} \varepsilon_{11}^{2} / 2+D_{112}^{\text {flock }} \varepsilon_{11}^{4} / 12+D_{120}^{\text {flock }} \varepsilon_{11} \varepsilon_{22}+D_{220}^{\text {flock }} \varepsilon_{22}^{2} / 2+ \\
& +D_{222}^{\text {flock }} \varepsilon_{22}^{4} / 12+D_{330}^{\text {flock }} \gamma_{12}^{2} / 2+D_{334}^{\text {flock }} \gamma_{12}^{6} / 30
\end{aligned}
$$

In the increments, a connection of the line forces through the increments of strains is written in the form:

$$
d\{N\}=[D] d\{\varepsilon\},
$$

where

$$
D_{11}=\frac{d^{2} W}{d \varepsilon_{11}^{2}}, D_{22}=\frac{d^{2} W}{d \varepsilon_{22}^{2}}, D_{33}=\frac{d^{2} W}{d \gamma_{12}^{2}}, D_{12}=\frac{d^{2} W}{d \varepsilon_{11} \varepsilon_{22}} .
$$

Then, according to (1), (3) the expressions for the stiffness characteristics of the nonflocked fabrics are obtained $D_{i j}^{f a b r}$ :

$$
\left\{\begin{array}{l}
D_{11}^{f a b r}=D_{110}+D_{112} \varepsilon_{11}^{2}, D_{22}^{f a b r}=D_{220}+D_{222} \varepsilon_{22}^{2} \\
D_{12}^{f a b r}=D_{120}, D_{21}^{f a b r}=D_{12}^{f a b r} \\
D_{33}^{f a b r}=D_{330}+D_{334} \gamma_{12}^{4} \\
D_{23}^{f a b r}=D_{32}^{f a b r}=D_{13}^{f a b r}=D_{31}^{f a b r}=0
\end{array}\right.
$$

The elastic law in the laboratory coordinate system $\tilde{x} \tilde{y}$, i.e. in the axes, being parallel to the edges of the test rectangular fabrics sample, shown in Fig. 1, takes the form:

$$
d\{\tilde{N}\}=[\tilde{D}] d\{\tilde{\varepsilon}\}, \quad[\tilde{D}]=[T][D][T]^{T}, \quad T=\left[\begin{array}{ccc}
\operatorname{Cos}^{2} \alpha & \operatorname{Sin}^{2} \alpha & -\operatorname{Sin} 2 \alpha \\
\operatorname{Sin}^{2} \alpha & \operatorname{Cos}^{2} \alpha & \operatorname{Sin} 2 \alpha \\
\frac{\operatorname{Sin} 2 \alpha}{2} & \frac{-\operatorname{Sin} 2 \alpha}{2} & \operatorname{Cos} 2 \alpha
\end{array}\right]
$$

For a numerical analysis of the strain process of the fabrics samples, the principle of Lagrange in the increments was used in the form:

$$
\int_{\Omega} \Delta N^{T} \delta \varepsilon d \Omega=\int_{\omega} \Delta p^{T} \delta u
$$

here $\Omega$ is an area occupied by a fabrics sample, $\omega$ is its boundary, $\Delta p$ is an increment of the vector of the line forces, applied at this boundary, $\delta \varepsilon, \delta \mathrm{u}$ are variations of the vectors of strains and movements. For a discretisation of the area, the FEM (finite element method) was used with a six-nodal triangular element of the second order [21].

For taking into account an inability of the fabrics to perceive the compressive load, the following approach was used. At each step of the load increment, the power field was analyzed $N_{i j}$. If along the basics or the weft, the efforts $N_{11}$ or $N_{22}$ took negative values, then at this step, according to the stiffness, $D_{11}$ or $D_{22}$ decreased by several orders of magnitude (in our case, 500 times). After that, a solution of the equation (7) was reconducted, and the efforts were recalculated. This procedure was repeated until the field of strains and efforts became stabilized.

\subsection{Method of determining the mechanical characteristics}

Let it be considered known the test findings of the structures with measurement of external influences, and the parameters of the mathematical model of behavior of the material and structures are the desired. The parameters of the models will be chosen so that the results of the numerical calculation and test findings were close based on a minimality of the quadratic residuality between the calculated and experimental results [22-24]: 


$$
\begin{aligned}
& \delta^{2}=\left.\left(v_{1}\left[(P)^{\exp }-(P)^{*}\right]^{2}+m_{1}\left[\Delta a^{\exp }-\Delta a^{*}\right]^{2}+k_{1}\left[\Delta b^{\exp }-\Delta b^{*}\right]^{2}\right)\right|_{\alpha_{1}}+ \\
& \left.\left(v_{2}\left[(P)^{\exp }-(P)^{*}\right]^{2}+m_{2}\left[\Delta a^{\exp }-\Delta a^{*}\right]^{2}+k_{2}\left[\Delta b^{\exp }-\Delta b^{*}\right]^{2}\right)\right|_{\alpha_{2}}+ \\
& +\left.\left(v_{n}\left[(P)^{\exp }-(P)^{*}\right]^{2}+m_{n}\left[\Delta a^{\exp }-\Delta a^{*}\right]^{2}+k_{n}\left[\Delta b^{\exp }-\Delta b^{*}\right]^{2}\right)\right|_{\alpha_{n}}
\end{aligned}
$$

Here $\mathrm{n}$ is a number of the performed, $\Delta a^{*}, \Delta b^{*}, \Delta a^{\exp }, \Delta b^{\exp }$ are calculated and experimental values of changes in the sides of the sample in the longitudinal and transverse directions, respectively (Fig. 1), $\alpha_{1}, \alpha_{2}, \ldots . \alpha_{n}$ are the angles between the base and the long side of the sample, $v_{1}, \ldots, v_{n}, m_{1}, . . m_{n}, k_{1}, \ldots k_{n}$ are the weight coefficients.

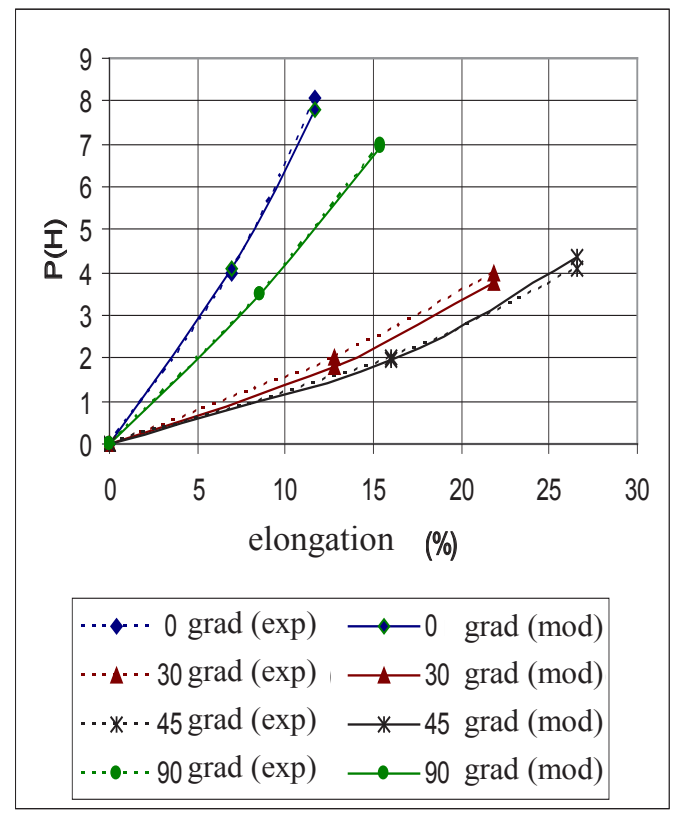

Fig. 2. Dependence of the average force at the end $\mathrm{P}$ on the elongation of the experimental data and the theoretical curve according to the obtained stiffness characteristics of the polyester tissue, subjected to a cold plasma treatment during $180 \mathrm{sec}$.

The stiffness characteristics $D_{110}, D_{114}, D_{220}, D_{224}, D_{120}, D_{330}, D_{334}$. are unknown. In this case, the following restriction must be fulfilled:

$$
D_{11} D_{22}-D_{12}^{2}>0
$$

The condition (9) will be satisfied if $D_{110} D_{220}-D_{120}^{2}>0$ is accepted. To find the minimum $\delta^{2}$ the standard gradient methods were used. The stiffness characteristics $D_{110}, D_{112}, D_{220}, D_{222}, D_{120}, D_{330}, D_{334}$ were determined for the both flocked and original fabricss, based on the data analysis of the sample tests, cut out at the different angles $\left(0^{0}, 90^{0}, 45^{0}, 30^{0}\right)$ to the base under different stretching forces.

In order to refine the techniques, the above approach was first tested on the following problem. A direct problem was solved with the given stiffness characteristics for the samples cut out at the different angles $0^{0}, 90^{\circ}, 45^{\circ}$. The results obtained were considered «experimental». Then a inverse problem was solved on the basis of the obtained «experiments», i.e. the stiffness characteristics of this mathematical model were 
determined. The results showed the efficiency of the techniques and the developed program.

\subsection{Verification of the techniques by the example of determining the stiffness characteristics for the fabrics subjected to the treatment with a low- temperature plasma}

Further, the results of the experiments were analyzed, where the polyester fabrics, treated during $180 \mathrm{sec}$. with a cold plasma, was considered as an experimental sample. A loading diagram of the sample is shown on Fig.1. An identification of the unknown $D_{220}, D_{222}, D_{120}, D_{330}, D_{334}$ was carried out according to the experimental data for the lowtemperature plasma-modified fabrics samples, cut out at the angles to the base $0^{0}, 90^{\circ}, 45^{\circ}$. The sample, cut out at the angle $30^{0}$ to the base, was considered a control one.

As a result, the following stiffness characteristics were obtained for the polyester fabrics treated with a cold plasma:

$$
\begin{aligned}
& D_{110}=1.2, D_{112}=5900, D_{220}=0.835, D_{222}=1000, \\
& D_{120}=0.178, D_{330}=0.045, D_{334}=2
\end{aligned}
$$

In (10), the stiffness coefficients have a number of dimensions [MPa* mm] .

Next, the dependence $P(\Delta a)$ was calculated for the angles $0^{0}, 90^{\circ}, 45^{\circ}$, as well as for the control sample with an angle of inclination to the base $\alpha=30^{\circ}$. The results are shown in Fig. 2. It is seen that the results obtained are in good agreement with the experiment.

\subsection{Determination of the stiffness characteristics for the original fabrics, fabrics with glue and flocked fabrics from the results of the stretching experiments}

This section presents the results of processing the experimental data for the original fabrics, fabrics with an adhesive layer and flocked fabrics in order to determine the stiffness characteristics of the fabrics, glue and flocked layer. The stiffness characteristics $D_{i j}$ for the fabrics were also taken in the form (5). For performing numerical calculations, the experiments were used carried out for the samples which were cut out at the angles $0^{0}, 90^{0}, 45^{0}, 30^{0}$. In contrast to the previous case, these experiments do not know the values $\Delta b$ - changes in the transverse dimensions of the sample. However, in this case, it is possible to determine 4 functions $D_{i j}$, but already based on the test results of 4 samples cut out at the different angles to the base $\alpha$.

After processing the experiments for the original fabrics, the following stiffness characteristics were obtained (fabrics thickness was $0.15 \mathrm{~mm}$, a number of dimensions $D_{i j k}$ - $\left.\left[\mathrm{MPa}^{*} \mathrm{~mm}\right]\right)$ :

$$
\begin{aligned}
& \left(D_{110}\right)^{f a b r}=8,\left(D_{112}\right)^{f a b r}=43000,\left(D_{220}\right)^{f a b r}=3.5,\left(D_{222}\right)^{f a b r}=3500, \\
& \left(D_{120}\right)^{f a b r}=0.97,\left(D_{330}\right)^{f a b r}=0.17,\left(D_{334}\right)^{f a b r}=195 .
\end{aligned}
$$

For the tissie with glue, the following results were obtained (the thickness of the raw glue was $0.2 \mathrm{~mm}$ ): 


$$
\begin{aligned}
& \left(D_{110}\right)^{\text {fabr }+ \text { qlue }}=21,\left(D_{112}\right)^{\text {fabr }+ \text { qlue }}=49000, \\
& \left(D_{220}\right)^{\text {fabr }+ \text { qlue }}=5.049,\left(D_{222}\right)^{\text {fabr }+q l u e}=4000, \\
& \left(D_{120}\right)^{\text {fabr }+ \text { qlue }}=0.97,\left(D_{330}\right)^{\text {fabr }+ \text { qlue }}=0.26, \\
& \left(D_{334}\right)^{\text {fabr }+ \text { qlue }}=235 .
\end{aligned}
$$

For the flocked fabrics it was found (the thickness of the flocked fabrics was $0.65 \mathrm{~mm}$ ):

$$
\begin{aligned}
& \left(D_{110}\right)^{\text {flock }}=26,\left(D_{112}\right)^{\text {flock }}=55500, \\
& \left(D_{220}\right)^{\text {flock }}=6,\left(D_{222}\right)^{\text {flock }}=4100, \\
& \left(D_{222}\right)^{\text {flock }}=4100,\left(D_{120}\right)^{\text {flock }}=0.97, \\
& \left(D_{330}\right)^{\text {flock }}=0.27,\left(D_{334}\right)^{\text {flock }}=235.5 .
\end{aligned}
$$

It was found that the experimental results and the curves, obtained according to the developed model, are in good agreement with each other. The residuals have the values about $10 \%$.

\subsection{Possibility of applying the theory of mixtures when making calculation of a flocked fabrics for stretching}

This section presents the results of a study of two approaches to solving the problem of stretching fabrics samples. In the first one, the theory of mixtures is used, and in the second one, a pattern configuration of the flocked part of the fabrics is taken into account. In this case, a hypothetical flocked fabrics was considered, in which the stiffness of the flocked part could be higher than that of the real one. This excess was determined by the coefficient $R=D^{\text {flock }} / D_{0}^{\text {flock }}$, where the components of the matrix $D_{0}^{\text {flock }}$ were determined by the relations (13).

The dependences of the force $P$ were determined, which is required for elongation of the sample by $20 \%$, on the fraction of the area of the flocked part of the fabrics at the different $\mathrm{R}$, obtained with an accurate account of the pattern configuration of the flocked part, and also obtained according to the theory of mixtures. In the latter case, it was assumed that:

$$
D_{i j}^{S}=D_{i j}^{f a b r}\left(1-A_{\text {flock }}\right)+D_{i j}^{\text {flock }} A_{\text {flock }},
$$

where $A_{\text {flock }}$ is a specific area of the flocked part.

An analysis of the numerical experiments shows that, in our case, the formula of mixtures for the practical calculations can be applied with an error of about $5 \%$, if the stiffnesses of the flocked part differ from the stiffness of the fabrics by no more than an order of magnitude.

\subsection{Dependence of the stiffness characteristics of flocked fabrics on the thickness of the raw glue}

Further, a possibility of using the theory of mixtures for averaging the stiffness characteristics with respect to thickness was studied. According to this theory, the components of the stiffness matrix of the glued fabrics can be represented as:

$$
D_{i j}^{f a b r+q l u e}=D_{i j}^{f a b r}+D_{i j}^{q l u e} .
$$

The adhesive coating was assumed to be isotropic: 


$$
\begin{aligned}
& D_{11}^{\text {qlue }}=D_{22}^{\text {qlue }}, D_{12}^{\text {qlue }}=D_{21}^{\text {qlue }}, \\
& D_{11}^{\text {qlue }}=E_{\text {qlue }} h^{\text {qlue }} /\left(1-v_{\text {qlue }}^{2}\right) \\
& D_{12}^{\text {qlue }}=v_{\text {qlue }} D_{11}^{\text {qlue }} .
\end{aligned}
$$

For the fabrics, the relations (4) were used.

As shown by processing of the experimental data in determining the stiffness of a fabrics composite material by averaging the stiffness of the fabrics and glue with respect to thickness, the theory of mixtures (15) does not allow determining the parameters $D^{\text {que }}$ with an acceptable accuracy from the discrepancy between the experimental and numerical results.

Therefore, to calculate stiffnesses of the flocked fabrics, depending on a thickness of the glue, it is necessary to take some other approaches. The simplest is a method of expanding these ratios in a series in terms of the degrees of a thickness of the raw glue $h^{\text {qlue }}$.

Since in the above experiments the stiffness characteristics were obtained only for two cases (for the initial fabrics and for the fabrics in which a thickness of the raw glue $h^{\text {qlue }}$ is $0.2 \mathrm{~mm}$ ), it is possible to use a polynomial of only the 1 st order.

Then the stiffness characteristics for the flocked fabrics can be represented as:

$$
\begin{aligned}
& D_{11}\left(h^{h^{q l u e}}, \varepsilon_{11}\right)=\left(8+43000 \varepsilon_{11}^{2}\right) \cdot \frac{0.2-h^{h^{\text {qlue }}}}{0.2}+\left(26+55500 \varepsilon_{11}^{2}\right) \cdot \frac{h^{h^{\text {qlue }}}}{0.2}, \\
& D_{22}\left(h^{h^{\text {qlue }}}, \varepsilon_{22}\right)=\left(3.5+3500 \varepsilon_{22}^{2}\right) \cdot \frac{0.2-h^{h^{q l u e}}}{0.2}+\left(6+4100 \varepsilon_{22}^{2}\right) \cdot \frac{h^{h^{q l u e}}}{0.2}, \\
& D_{33}\left(h^{h^{\text {qlue }}}, \gamma_{12}\right)=\left(0.17+194.5 \gamma_{12}^{4}\right) \cdot \frac{0.2-h^{h^{q l u e}}}{0.2}+\left(0.27+235.5 \gamma_{12}^{4}\right) \cdot \frac{h^{h^{q l u e}}}{0.2}, \\
& D_{12}\left(h^{h^{\text {qlue }}}\right)=0.978 \cdot \frac{0.2-h^{h^{\text {qlue }}}}{0.2}+0.978 \cdot \frac{h^{h^{\text {qlue }}}}{0.2},
\end{aligned}
$$

where $h^{\text {qlue }}$ is a thickness of the raw glue.

\section{Discussions}

The developed techniques for determining the stiffness characteristics of the flocked fabrics, based on the results of testing the samples, cut off at the different angles $0^{0}, 90^{0}, 30^{0}, 45^{0}$ to the base under different stretching forces, made it possible to analyze the effect of flocking on its mechanical characteristics. It was revealed that the theory of mixtures, when averaging the properties of the fabrics and glue with respect to thickness, does not allow determining the stiffness characteristics with acceptable accuracy. The limits of applicability of the theory of mixtures were determined when carrying out averaging of the mechanical characteristics with respect to the area of the flocked fabrics.

\section{Acknowledgements}

The work was performed within the framework of the Russian Foundation for Basic Research (project No. 19-08-00349) and the Russian Science Foundation (project No. 19-19-00059). 


\section{References}

1. V. V. Khammatova, Influence of the multipacting plasma on the structure and physical and mechanical properties of textile fibers, Chemical fibers, 4, 47-49 (2005)

2. M. N. Serazutdinov, R. A. Kayumov, Calculation of the strain properties of textile materials, News of universities. Technology of Textile industry, 5, 34-38 (2006)

3. I. Sh. Abdullin, V. V. Khammatova, Influence of low-temperature plasma flow on the properties of textile materials (Publish. house of Kazan University, Kazan, 2003)

4. R. A. Kayumov, I. Sh. Abdullin, M. N. Serazutdinov, V. V. Khammatova, I. Z. Mukhamedova, E. V. Kumpan, A. M. Shakirova, Physically nonlinear model of the fabrics materials processed in a high-frequency plasma flow, Bulletin of Kazan Technological University, 6, 42-53 (2010)

5. I. Sh. Abdullin, V. V. Khammatova, E. V. Kumpan, Experimental studies of the influence of the multipacting plasma on the adhesive properties of composite materials, Applied Physics, 6, 92-94 (2005)

6. M. F. Shaekhov, V. I. Khristolyubova, A. A. Rushintsev, V. E. Gorelysheva, Study of the characteristics of the high-frequency discharge flow of low pressure in technological processes of the diffusion formation of coatings on the products of complex configuration, Bulletin of the Technological University, 19(13), 98-100 (2016)

7. I. Besshaposhnikova, S. E. Artemenko, L. G. Panova, T. V. Kulikova, O. A. Grishina, V. A. Steinle, M. V. Zagoruiko, Fire retardant modification of synthetic materials under the influence of laser radiation, Chemical fibers, 1, 48-51 (2008)

8. W. N. W Ismail. Sol-gel technology for innovative fabric finishing - A Review, J SolGel Sci. Technol, 78, 698-707 (2016)

9. J. Foksowicz-Flaczyk, J. Walentowska, M.Przybylak, H. Maciejewski. Multifunctional durable properties of textile materials modified by biocidal agents in the sol-gel process, Surf. Coat. Technol, 304, 160-166 (2016) DOI:10.1016/j.surfcoat.2016.06.062

10.X. Guo, Q. Zhang, X. Ding, Q. Shen, C. Wu, L. Zhang, H.Yang. Synthesis and application of several sol-gel-derived materials via sol-gel process combining with other technologies: a review, Sol-Gel Sci. Technol, 79, 328-358 (2016) DOI: 10.1007/s10971-015-3935-6

11.M. Grace, N. Chand, S. Kumar Bajpai. Copper Alginate-Cotton Cellulose (CACC) Fibers with Excellent Antibacterial Properties, Journal of Engineered Fibers and Fabrics, 4, 24-35 (2009) DOI: 10.1177/155892500900400303

12.H. E. Emam, A. P. Manian, B. Siroka, H. Duelli, P. Merschak, B. Redl, Th. Bechtold. Copper (I) oxide surface modified cellulose fibers-Synthesis, characterization and antimicrobial properties, Surface \& Coatings Technology, 254, 344-351 (2014) DOI: 10.1016/j.surfcoat.2014.06.036

13. A. Burkitbay, B.R. Taussarova. A.Z. Kutzhanova, S.M. Rakhimovu. Development of a Polymeric Composition for Antimicrobial Finish of Cotton Fabrics, Fibers \& Textiles in Eastern Europe, 2(104), 96-101 (2014)

14.B. R. Tausarova, S. M. Rakhimova. Cellulosic materials with antibacterial properties modified with copper nanoparticles, Chemistry of plant raw materials, 1, 163-169 (2018)

15. A. G. Hassabo, M. E. Kl-Naggar, A. L. Mohamed, A. Ali, A.A. Hebeish Development of multifunctional modified cotton fabric with tricomponent-nanoparticlcs of silver, copper and zincoxide, Carbohydrate Polymers, 210, 144-156 (2019)

16.Q. Xu, X. Ke, N. Ge, L. Shen, Y. Zhang, F. Fu, X. Liu. Preparation of Copper Nanoparticles Coated Cotton Fabrics with Durable Antibacterial Properties, Fibers and Polymers, 19, 1004-1013 (2018) DOI: 10.1007/s12221-018-8067-5 
17.V.A. Semenov. Theory and practice of electroflocking (Publishing house of VZPI, Moscow, 1992)

18.L. V. Lobova, N. E. Bershev, I. A. Khromeeva. Superinsulating flocked material, Technical textiles, 2, 33-35 (2001)

19.O. M. Ivanov, E. N. Bershev. Determination of the limiting characteristics of the flocking process, Electronic processing of materials, 4, 34-37 (1988)

20.E. H. Bershev, O. M. Ivanov, I.O. Red'kin. Comparison of the efficiency of flock application on textile material during electroflocking by various methods, News of universities. Ser. Technology of Textile industry, 4, 35-38 (1987)

21.I. F. Obraztsov, L. M. Saveliev, Kh.S. Khazanov. Finite Element Method in Problems of Aircraft Structural Mechanics (Higher school, Moscow, 1985)

22.R. A. Kayumov, R. O. Nezhdanov, B.F. Tazyukov. Determination of the characteristics of fibrous composite materials by identification methods (Publishing house of KSU, Kazan, 2005)

23.V. G. Nizameev, F. F. Basharov. The method of optimization of design parameters of truss plates for profiled flooring, Science prospects 7(106), 81-87 (2018)

24.V. G. Nizameev, F. F. Basharov. The study of the bearing capacity of profiled flooring with the truss system, Science and business development ways 6(84), 76-81 (2018) 